\title{
Viewpoint
}

\section{A breakthrough observation for neutron dripline physics}

\author{
Kirby W. Kemper and Paul D. Cottle \\ Physics Department, Florida State University, Tallahassee, Florida 32306, USA
}

Published February 8, 2010

Observation of a two-neutron halo in ${ }^{22} \mathrm{C}$ makes it the heaviest "Borromean" nucleus yet observed.

\author{
Subject Areas: Nuclear Physics
}

\author{
A Viewpoint on: \\ Observation of a Large Reaction Cross Section in the Drip-Line Nucleus ${ }^{22} \mathrm{C}$ \\ K. Tanaka et al. \\ Phys. Rev. Lett. 104, 062701 (2010) - Published February 8, 2010
}

\begin{abstract}
The neutron halo is one of the most spectacular phenomena that nuclear structure physicists have observed in their exploration of the isotopes at and close to the neutron dripline (the curve on a plot of atomic number versus neutron number beyond which neutron-rich nuclei begin "leaking" neutrons) using exotic beam facilities. Atomic nuclei are usually uniformly dense objects with surfaces that are nearly well defined, having only a modest amount of diffuseness. However, in halo nuclei one or more nucleons have wave functions that extend far outside the nucleus so that the matter distribution has a long tail.
\end{abstract}

The first halo nucleus observed was ${ }^{11} \mathrm{Li}$ [1], which has two correlated neutrons in its halo [2]. The experiment that resulted in the observation of the halo was performed at Lawrence Berkeley Laboratory with the Bevalac, the only relativistic heavy-ion accelerator in the world in the mid 1980s. Researchers slammed a high-energy beam of the stable isotope ${ }^{20} \mathrm{Ne}$ into a "production target," and then separated the short-lived products of that "fragmentation" reaction using magnets mounted on the beam line. The resulting shortlived (half-life $8.6 \mathrm{~ms}$ ) ${ }^{11} \mathrm{Li}$ nuclei were then steered onto targets of beryllium, carbon, and aluminum and the cross-sections for these resulting reactions measured.

The radius of a nucleus as a function of atomic mass number $A$ can typically be calculated as $(1.2 \mathrm{fm}) A^{1 / 3}$, which for ${ }^{11} \mathrm{Li}$ would give $2.7 \mathrm{fm}$. The initial analysis of the data of Tanihata et al. gave a large rms matter radius of $3.11 \pm 0.16 \mathrm{fm}$, but refinements to the reaction model eventually resulted in a conclusion that the rms radius is even larger $(3.53 \pm 0.10 \mathrm{fm}$ [3]). Weak binding is critical to the formation of a halo, and with a two-neutron separation energy of $300 \pm 19 \mathrm{keV},{ }^{11} \mathrm{Li}$ provided the prototypical example of this as well. Finally, ${ }^{11} \mathrm{Li}$ essentially consists of three pieces-two neutrons and a ${ }^{9} \mathrm{Li}$ "core"- that all must be present for the system to be bound since ${ }^{10} \mathrm{Li}$ is unbound. Such a three-body quan- tum system where all three parts must be present for the existence of the system is called "Borromean" after the three interlocking rings on the 15th century coat of arms of the Borromeo family in northern Italy (Fig. 1). The three rings are connected in such a way that the cutting of one ring results in the separation of all three.

With advances in the production of exotic beams that have involved both improvements in the accelerators for the "primary" stable beams and the development of highly sophisticated magnetic spectrometers to separate the exotic products of the fragmentation reactions between the stable beams and the production targets, detailed studies of dripline isotopes of heavier elements have become possible. As K. Tanaka and colleagues from several institutions in Japan now report in Physical Review Letters, the Radioactive Isotope Beam Factory at RIKEN [4], which delivered its first beam in 2006, has now been used to measure a two-neutron halo in the dripline nucleus ${ }^{22} \mathrm{C}$ [5].

The sensitivity of the measurement is impressive, having been performed with a beam rate of only ten ${ }^{22} \mathrm{C}$ nuclei per hour. With twice as many protons and neutrons as ${ }^{11} \mathrm{Li},{ }^{22} \mathrm{C}$ is the heaviest Borromean nucleus yet observed. The rms matter radius of $5.4 \pm 0.9 \mathrm{fm}$ deduced for ${ }^{22} \mathrm{C}$ differs markedly from the "standard" nuclear radius of $(1.2 \mathrm{fm}) A^{1 / 3}=3.4 \mathrm{fm}$. Furthermore, the cross section for the ${ }^{22} \mathrm{C}$ breaks sharply from the trend exhibited by the measurements of lighter carbon isotopes that Tanaka et al. also performed.

As ${ }^{11} \mathrm{Li}$ is loosely bound, so is ${ }^{22} \mathrm{C}$ (although further mass measurements on this nucleus are certainly called for-Tanaka et al. quote a value of $420 \pm 940 \mathrm{keV}$ ). The authors also argue, on the basis of the systematic behavior of interaction cross sections for the heavy carbon isotopes, that the two halo neutrons preferentially occupy the $1 s_{1 / 2}$ orbit in the $s d$ shell. This is in some contrast to the situation in ${ }^{11} \mathrm{Li}$, where the contribution to the ground-state wave function of the halo neutrons is 

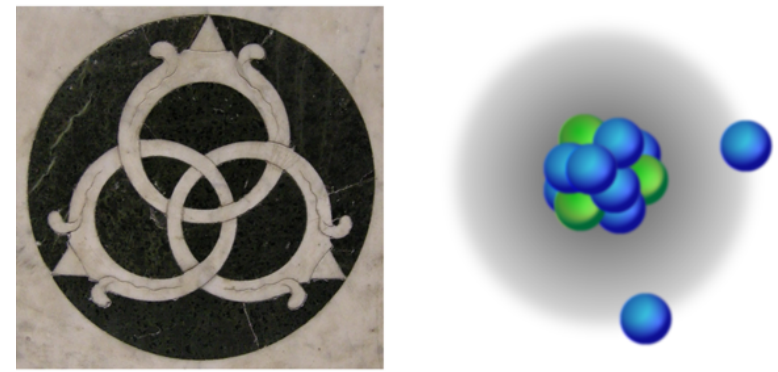

FIG. 1: ${ }^{22} \mathrm{C}$ is now the heaviest observed Borromean nucleus. Borromean nuclei are named after the rings from the 15th century crest of the Borromeo family from northern Italy. The rings are connected in such a way that the cutting of one ring results in the separation of all three. (Left) Marble representation of the Borromean rings, used as an emblem of Lorenzo de Medici in San Pancrazio, Florence [13]. (Right) Schematic view of ${ }^{22} \mathrm{C}$ showing the two halo neutrons around a core. Removing any one element makes the entire structure unstable. (Illustration:Alan Stonebraker)

divided approximately equally between the $0 p^{2}{ }_{1 / 2}$ and $1 s^{2}{ }_{1 / 2}$ shell model configurations [6].

In the case of ${ }^{11} \mathrm{Li}$, the large role played by the $1 s_{1 / 2}$ neutron orbit signals the disappearance of the $N=8$ major shell closure that exists in stable nuclei. The shifting of shell closures near the neutron dripline is a major theme of studies of exotic nuclei, with such shifts occurring systematically in neutron-rich isotopes (for example, see Refs. [7, 8]). Such shifts also play an important role in the formation of the neutron halo in ${ }^{22} \mathrm{C}$ : The presence of an $N=16$ shell closure in neutron-rich isotopes, recently highlighted by the determination of the doubly-closed-shell nature of the neighboring isotone ${ }^{24} \mathrm{O}[9,10]$, drives the purity of the ground-state wave function of the neutron halo in ${ }^{22} \mathrm{C}$.

In fact, a comparison of the three $N=16$ dripline nuclei- ${ }^{22} \mathrm{C}^{23} \mathrm{~N}$, and ${ }^{24} \mathrm{O}$ - provides a beautiful illustration of the core dynamic of nuclear structure physics: the sensitive dependence of nuclear behavior on proton and neutron numbers. Despite the presence of the $N=16$ shell closure in all three of these isotones, their geometries are quite different. ${ }^{22} \mathrm{C}$ is weakly bound and, as Tanaka et al. have shown, possesses a clear neutron halo (rms radius of $5.4 \pm 0.9 \mathrm{fm}$ ). ${ }^{23} \mathrm{~N}$ and ${ }^{24} \mathrm{O}$ are much more tightly bound-the single neutron separation energies in both ${ }^{23} \mathrm{~N}$ and ${ }^{24} \mathrm{O}$ are several $\mathrm{MeV}$ - and Ozawa et al.[11] showed that their matter radii are much closer to the "normal" nuclear values of $(1.2 \mathrm{fm}) A^{1 / 3}$ (the radii reported in Ref. [11] are $3.41 \pm 0.23 \mathrm{fm}$ and $3.19 \pm 0.13 \mathrm{fm}$ for ${ }^{23} \mathrm{~N}$ and ${ }^{24} \mathrm{O}$, respectively, while the corresponding values of $(1.2 \mathrm{fm}) A^{1 / 3}$ are $3.41 \mathrm{fm}$ and $\left.3.46 \mathrm{fm}\right)$. In fact, while ${ }^{22} \mathrm{C}$ has now been shown to be one of the most exotic of the 3000 known isotopes, the neighboring isotone ${ }^{24} \mathrm{O}$ has proven to display, in many respects, the behavior of a conventional doubly-magic nucleus $[9,10]$ (even if the $N=16$ shell closure is exotic).

In this context, it is worth noting that at only one proton above ${ }^{24} \mathrm{O}$, the neutron dripline jumps from $N=$ 16 to $N=22$ (the heaviest bound fluorine isotope is ${ }^{31} \mathrm{~F}$ [12]). This phenomenon continues to challenge our understanding of nuclear behavior in extreme conditions. In short, the discovery of a neutron halo in ${ }^{22} \mathrm{C}$ by Tanaka et al.[5] is not just a tour de force of the application of frontier experimental techniques to the search for exotic nuclear behavior. When taken together with the recent advances in the understanding of the neighboring isotone ${ }^{24} \mathrm{O}$, the ${ }^{22} \mathrm{C}$ measurement provides a critical step forward. Detailed studies of heavier isotopes along the neutron dripline will become possible during the next decade as new exotic beam facilities and more sensitive detectors come online. These studies will reveal new halo nuclei, novel shell effects, and perhaps even phenomena that are completely unexpected in our understanding of the basic physics of the neutron dripline.

\section{Acknowledgments}

This work was supported by the National Science Foundation and the State of Florida.

\section{References}

[1] I. Tanihata, H. Hamagaki, O. Hashimoto, Y. Shida, N. Yoshikawa, K. Sugimoto, O. Yamakawa, T. Kobayashi, and N. Takahashi, Phys. Rev. Lett. 55, 2676 (1985).

[2] P. G. Hansen and B. Jonson, Europhys. Lett. 4, 409 (1987).

[3] J. S. Al-Khalili and J. A. Tostevin, Phys. Rev. Lett. 76, 3903 (1996).

[4] T. Motobayashi and Y. Yano, Nuclear Physics News 17, Issue 4, 5(2007).

[5] K. Tanaka et al., Phys. Rev. Lett. 104, 062701 (2010).

[6] H. Simon et al., Phys. Rev. Lett. 83, 496 (1999).

[7] J. Fridmann et al., Nature (London) 435, 922 (2005).

[8] B. Bastin et al., Phys. Rev. Lett. 99, 022503 (2007).

[9] C. R. Hoffman et al., Phys. Lett. B 672, 17 (2009).

[10] R. Kanungo et al., Phys. Rev. Lett. 102, 152501 (2009).

[11] A. Ozawa et al., Nucl. Phys. A 691, 599 (2001).

[12] H. Sakurai et al., Phys. Lett. B 448, 180 (1999).

[13] Photograph by Sailko, http://commons.wikimedia. org/wiki/File:Tempietto,_formelle_18_impresa_ personale_di_lorenzo_de $\backslash \% 27$ medici.JPG; See also http://www.liv.ac.uk/ spmr02/rings/medici.html. 


\section{About the Authors}

\section{Kirby W. Kemper}

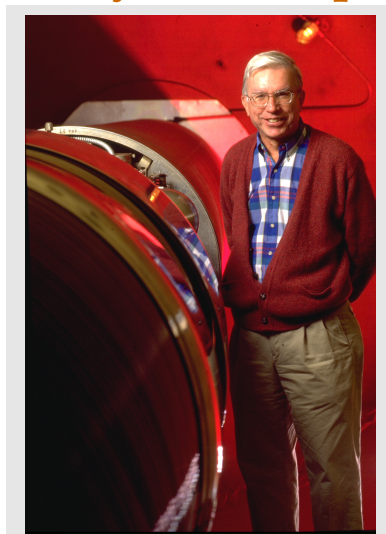

Kirby Kemper received his B.S. in physics from Virginia Tech in 1962 and his Ph.D. in experimental nuclear physics from Indiana University in 1968. His thesis topic involved the study of beta decay angular correlations. He was then hired as a postdoc in the accelerator laboratory at Florida State University. He obtained a faculty position at Florida State in 1971 and has been on the faculty since then. He presently serves as the Vice President for Research. He has carried out nuclear scattering and reaction experiments in low-energy nuclear physics at FSU, Argonne National Laboratory, the Australian National University, Oak Ridge National Laboratory, Notre Dame University, the National Superconducting Cyclotron Laboratory at MSU, GANIL in France, and the Heavy Ion Lab in Warsaw, Poland. His current interest centers around the study of loosely bound light nuclei as well as nuclei close to the neutron dripline.

\section{Paul D. Cottle}

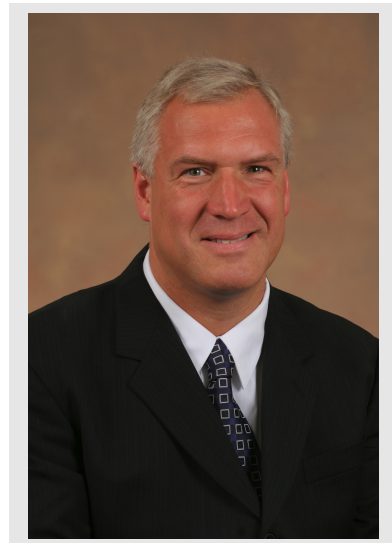

Paul Cottle received his Ph.D. in physics from Yale in 1986 for a study of high spin behavior in ${ }^{219,220} \mathrm{Ra}$ and ${ }^{216} \mathrm{Rn}$. He joined the Florida State University Physics faculty that year and has continued there since then. He was named an NSF Presidential Young Investigator in 1987. His research presently focuses on the structure of exotic nuclei and includes experiments at both FSU's Fox Superconducting Linear Accelerator Laboratory and the National Superconducting Cyclotron Laboratory at Michigan State University. He is serving as Chair of the Southeastern Section of the American Physical Society. 